\title{
General Paresis in a Child with Unusual Presentation
}

Muhamad Azamin Anuar, Wan Alina Khadijah, Asrar Abu Bakar, Mossad Abdelhak Shaban Mohamed, Taufiq Hidayat Hasan

Kulliyyah of Medicine, International Islamic University Malaysia, Kuantan, Pahang.

Introduction: Acute onset quadriparesis can be a manifestation of a variety of neurological, metabolic or autoimmune conditions. Rarely, it could be one of many clinical presentations of juvenile dermatomyositis which belongs to the group of idiopathic inflammatory myopathies of childhood. We report the case of a 9-year old girl who presented with global myopathy over a two-month period. Case report: A 9-year-old girl referred with a 2 months history of generalised muscular pain and weakness. There were no significant history of preceded illness, trauma or excessive strenuous exercises. She had no other systemic complaints such as fever or skin rash. Past medical history was unremarkable except for recurrent tonsillitis. Physical examination revealed a child with normal alertness and behaviour. She has notable generalised swelling of all four limbs. Her neurological examination revealed normal tone however her power was definitely reduced in all four limbs (Graded 3/5). She demonstrated signs of proximal myopathy. Subsequent investigations revealed high Creatinine Kinase (CK) levels of $6470 \mathrm{U} / \mathrm{L}$, ESR $84 \mathrm{~mm} / \mathrm{hr}$ with deranged transaminases and positive serum mycoplasma and CMV IgM. Her MRI brain and spine along with lumbar puncture results were normal. She was referred to tertiary centre for further evaluation as her weakness progressed. An MRI muscle demonstrated marked inflammation in all four limbs including paravertebral muscles. Her muscle biopsy showed inflammatory myopathy leading to a diagnosis of juvenile dermatomyositis (JDM). She is now showing sustained clinical improvements following a course of immunoglobulin and corticosteroids therapy. Discussion: The diagnosis of JDM is generally considered in patients with rash-associated muscle weakness. Essentially, it involves assessment of muscle, skin, lung and cardiac involvement on top of baseline list of investigations that has been outline by Single Hub and Access point for pediatric Rheumatology in Europe (SHARE). This case reflects that JDM is possible despite non-existent of skin involvement. 